\title{
Invisibility of a Rectangular Prism Made of Anti-Isorefractive DNG Metamaterial Immersed in a Standing-Wave Field
}

\author{
Piergiorgio L. E. Uslenghi
}

\begin{abstract}
Scattering by a prism of rectangular cross-section made of double-negative metamaterial is considered, in the phasor domain. The prism is immersed in a standing-wave field formed by two plane waves of the same amplitude and polarization, propagating in opposite directions. Under two conditions relating the prism dimensions, the wavelength and the direction of incidence of the primary waves, the prism does not perturb the incident field, and geometrical optics provides the exact solution to the boundary-value problem.
\end{abstract}

\section{Introduction}

In many applications, it is desirable to blunt scattering by sharp edges. In the case of metallic (PEC) structures, this objective can be achieved either by using absorbers or by impinging upon the edge with several plane waves $[1,2]$ of appropriate amplitude, phase, polarization, and direction of incidence. If the scattering structure has characteristic dimensions, additional conditions involving such dimensions and the wavelength must also be imposed [2]. When scattering by edges is eliminated, the structure is immersed in standing-wave fields, and geometrical optics (GO) provides the exact solution to the boundary-value problem. For example, the 2D scattering by a PEC cylinder of rectangular cross section requires four incident plane waves for $\mathrm{GO}$ to be the exact solution [3]. An exact GO solution exists for some structures involving penetrable wedges (see, e.g., [4-7]). However, the only known exact GO solution for a single wedge under incidence by a single plane wave is scattering by a right-angle wedge made of antiisorefractive double-negative (DNG) metamaterial [8].

The behavior of the electromagnetic field in the vicinity of the edge of a penetrable wedge is well understood (see, e.g., $[9,10,11])$. In order to avoid scattering by the edge and obtain an exact GO solution, it is necessary not only to satisfy the boundary conditions on the faces of the wedge but also to avoid field discontinuities across all optical boundaries; this latter objective usually requires the presence of more than one incident plane wave.

In the present work, a prism of infinite length and rectangular cross section is considered. The prism is made of a lossless metamaterial that is anti-isorefractive to the surrounding medium, meaning that the intrinsic

Manuscript received 21 May 2021.

Piergiorgio L. E. Uslenghi is with the Department of Electrical and Computer Engineering, University of Illinois at Chicago, USA; e-mail: uslenghi@uic.edu. impedance is the same inside and outside the prism while the refractive indexes have opposite sign, as is required by causality [12]. On the assumption that the four edges of the prism do not scatter (to be verified a posteriori), a plane wave incident on a face of the prism is totally transmitted as though it were incident on an infinite plane, and reemerges into the surrounding medium after a second total transmission through another face. If only one wave is incident, this process gives rise to field discontinuities across optical boundaries and therefore cannot lead to an exact solution. However, if two plane waves of equal amplitude, polarization, frequency, and appropriate phases but propagating in opposite directions are incident on the prism, all field discontinuities across optical boundaries disappear and the GO field represents the exact solution; thus, the presence of the prism does not perturb the standing-wave field that would be present in the absence of the prism. This situation is possible if two relations involving the dimensions of the rectangular cross section, the wavelength of the incident waves, and the angle of incidence are satisfied. These two relations are those that would occur if the prism were made of a PEC material, but in that case four incident waves would be needed to avoid edge scattering $[2,3]$.

The geometry of the problem is shown in Section 2. The solution and the conditions under which it is valid are derived in Section 3. The exact GO solution presented herein constitutes a novel canonical solution of a boundary-value problem, and may be of practical interest in the validation of computer solvers.

The $2 \mathrm{D}$ analysis performed in this work may be extended to oblique incidence of the two primary waves with respect to the edges of the prism, by utilizing the general results derived in $[13,14]$.

\section{Geometry of the Problem}

A cross section of the DNG prism in any plane $z=$ constant is shown in Figure 1; it occupies the rectangle (0 $\leq x \leq a ;-b \leq y \leq 0)$ in the $(x, y)$ plane; without loss of generality, it is assumed that $a \leq b$. The prism is surrounded by a linear, isotropic, and lossless doublepositive (DPS) medium characterized by real positive permittivity $\varepsilon$ and permeability $\mu$ or, alternatively, by a real positive wave number $k=\omega \sqrt{\epsilon \mu}$ and a real positive intrinsic impedance $Z=Y^{-1}=\sqrt{\mu / \epsilon}$. The material inside the prism is a linear, isotropic, and lossless antiisorefractive DNG metamaterial characterized by real negative permittivity $-\varepsilon$ and permeability $-\mu$ or, alternatively, by a real negative wave number $-k$ and a real positive intrinsic impedance $Z$. 


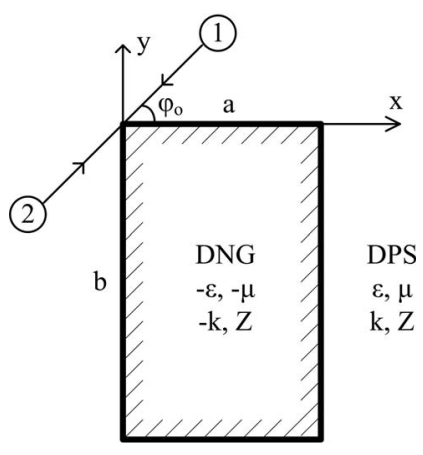

Figure 1. Geometry of the problem.

A first primary plane wave propagates in a direction that forms the angle $\varphi_{0}$ with the negative $x$ axis and the angle $\pi / 2-\varphi_{0}$ with the negative $y$-axis, where $0 \leq \varphi_{0} \leq \pi / 2$. A second primary plane wave propagates in the direction opposite to that of the first wave. The two incident waves are in phase at the edge $(x=y=0)$ of the prism and have the same amplitude, frequency, and polarization. The electric field is assumed to be parallel to the $z$-axis (E-polarization); the results for H-polarization are obtained easily by
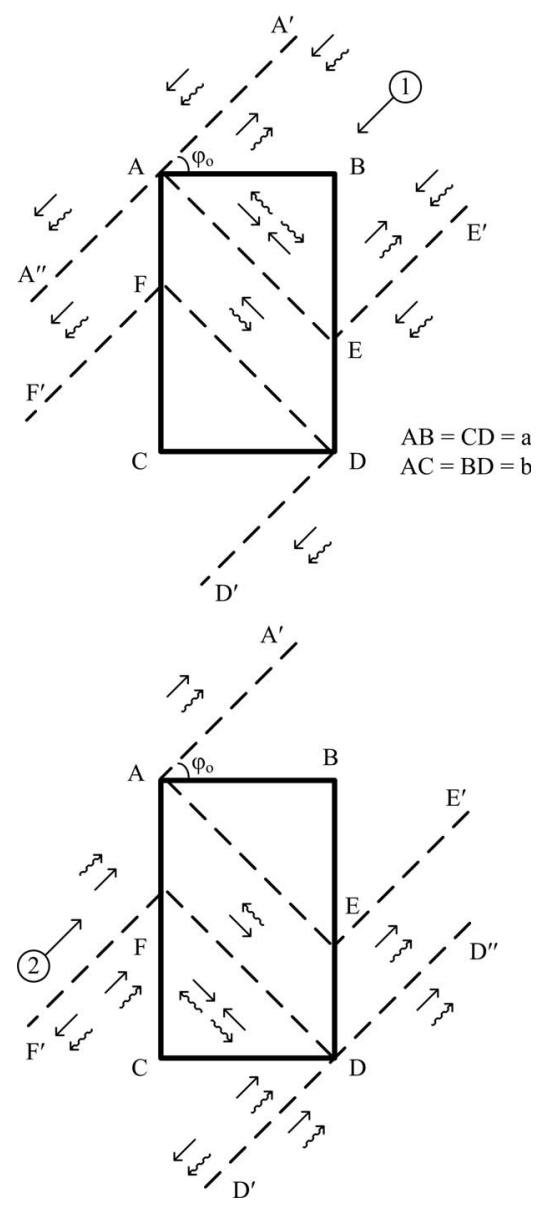

Figure 2. GO fields and optical boundaries when $\tan \varphi_{0}<b / a$.
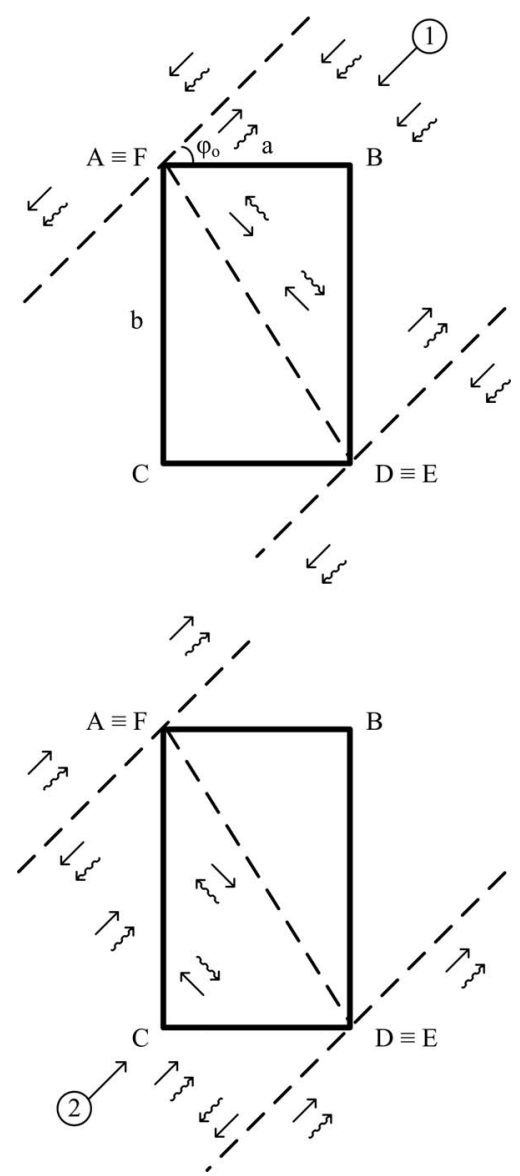

Figure 3. GO fields and optical boundaries when $\tan \varphi_{0}=b / a$.

duality. With the time-dependence factor $\exp (+j \omega t)$ omitted, the incident electric fields of the two waves are

$$
\underline{E}_{2}^{i}=\hat{z} e^{ \pm j k \Phi(x, y)}
$$

where

$$
\Phi(x, y)=x \cos \varphi_{0}+y \sin \varphi_{0}
$$

\section{Exact GO Solution}

The GO fields produced separately by each of the two incident waves are shown in Figure 2 when $\varphi_{0}<\arctan (b / a)$, in Figure 3 when $\varphi_{0}=\arctan (b / a)$, and in Figure 4 when $\varphi_{0}>\arctan (b / a)$. The directions of energy propagation and phase increase are indicated with straight and wavy arrows, respectively; the two directions are co-parallel in the DPS medium and antiparallel in the DNG material. Under incidence by one plane wave only, optical boundaries occur (shown as dashed lines), across which the GO field is discontinuous. For example, in the top part of Figure 2, the volume $\mathrm{A}^{\prime} \mathrm{AEE}^{\prime}$ is filled with a standing plane wave, the volume $\mathrm{F}^{\prime} \mathrm{FDD}^{\prime}$ has zero fields, and the remaining space is occupied by a single plane wave. Similar considerations apply to the bottom part of Figure 2 and to Figures 3 and 4. However, the 

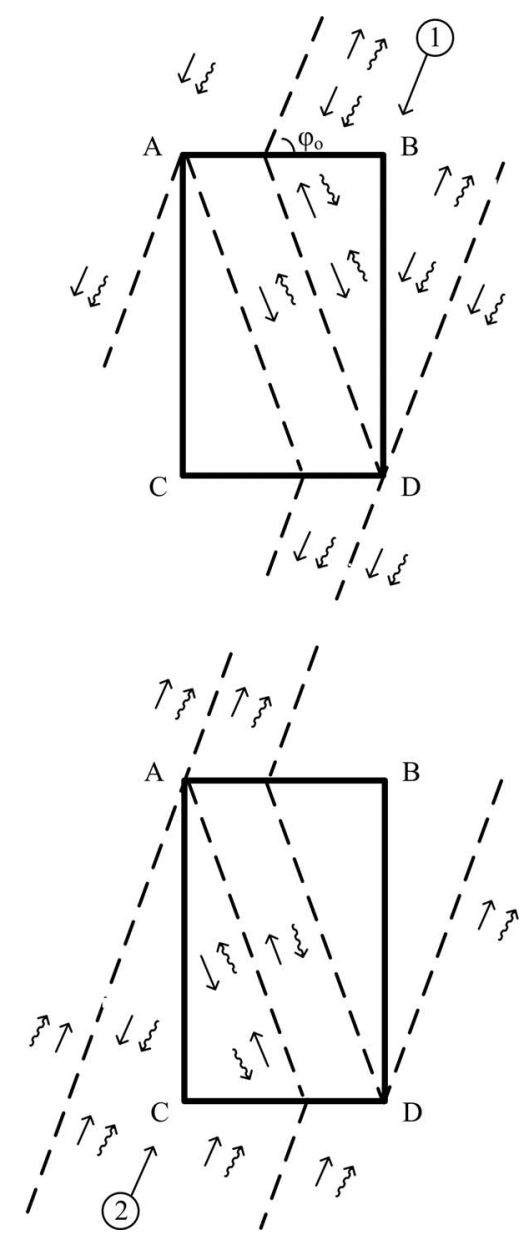

Figure 4. GO fields and optical boundaries when $\tan \varphi_{0}>b / a$.

discontinuities across all optical boundaries vanish under incidence by both plane waves, provided that the two conditions

$$
\begin{aligned}
& k a \cos \varphi_{0}=m \pi \\
& k b \sin \varphi_{0}=n \pi
\end{aligned}
$$

are satisfied, where $m$ and $n$ are integers.

The physical meaning of (3) and (4) is that each incident plane wave must have the same phase $(\bmod 2 \pi$ rad) at all three illuminated edges of the prism. Conditions (3) and (4) imply that the angle of incidence $\varphi_{0}$ and the wavelength $\lambda$ must satisfy the relations

$$
\begin{aligned}
\tan \varphi_{0} & =\frac{n a}{m b} \\
\lambda & =\frac{2 \pi}{k}=2 / \sqrt{\left(\frac{m}{a}\right)^{2}+\left(\frac{n}{b}\right)^{2}}
\end{aligned}
$$

Hence, for given $a$ and $b, \varphi_{0}$ depends only on the ratio $n / m$, whereas $\lambda$ depends on both $m$ and $n$. The frequency must be sufficiently high for GO to be the exact solution, and the maximum allowed values for the integers $m$ and $n$ must satisfy

$$
m_{\max } \leq 2 a / \lambda, \quad n_{\max } \leq 2 b / \lambda
$$

Under (3) and (4), the total GO field outside the prism is a standing wave with components

$$
\begin{aligned}
& E_{z}^{\mathrm{DPS}}=2 \cos k \Phi(x, y) \\
& H_{y}^{\mathrm{DPS}}=\mp 2 j Y\left\{\begin{array}{c}
\sin \varphi_{0} \\
\cos \varphi_{0}
\end{array}\right\} \sin k \Phi(x, y)
\end{aligned}
$$

whereas the total GO field inside the prism is a standing wave with components

$$
\begin{aligned}
E_{z}^{\mathrm{DNG}} & =2 \cos k \Phi(x,-y) \\
H_{y}^{\mathrm{DNG}} & =-2 j Y\left\{\begin{array}{l}
\sin \varphi_{0} \\
\cos \varphi_{0}
\end{array}\right\} \sin k \Phi(x,-y)
\end{aligned}
$$

The GO fields (8)-(11) satisfy Maxwell's equations and the boundary conditions on the four faces of the prism. The edges of the prism do not scatter, and (8)-(11) constitute the exact GO solution to the boundary-value problem.

\section{Acknowledgment}

The author is grateful to Baker B. Al-Bahri for typing the manuscript.

\section{References}

1. P. L. E. Uslenghi, "Exact Geometrical-Optics Scattering by a Class of Metallic Wedges Under Multiple PlaneWave Illumination, " Radio Science Letters, 1, 2019, doi: 10.46620/19-0005.

2. B. B. Al-Bahri and P. L. E. Uslenghi, "Exact Scattering by Metal Structures With Strips and Right-Angle Wedges," Radio Science Letters, 2, 2020, doi: 10. 46620/20-0022.

3. P. L. E. Uslenghi, "Exact Geometrical Optics Scattering by a Truncated Metallic Cylinder of Rectangular Cross Section Under Multiple Plane Waves Illumination," Photonics \& Electromagnetics Research Symposium, Rome, Italy, June 17-20, 2019, pp. 17-20.

4. P. L. E. Uslenghi, "Exact Solution for a Penetrable Wedge Structure," IEEE Transactions on Antennas and Propagation, 45, 1, January 1997, p. 179.

5. P. L. E. Uslenghi, "Exact Geometrical Optics Solution for an Isorefractive Wedge Structure," IEEE Transactions on Antennas and Propagation, 48, 2, February 2000, pp. 335336.

6. P. L. E. Uslenghi, "Exact Geometrical Optics Scattering From a Tri-Sector Isorefractive Wedge Structure," IEEE Antennas and Wireless Propagation Letters, 3, 2004, pp. 94-95.

7. P. L. E. Uslenghi, "Exact Geometrical Optics Scattering From a Right-Angle Isorefractive Wedge Structure," IEEE Antennas and Wireless Propagation Letters, 3, 2004, pp. 127-128.

8. P. L. E. Uslenghi, "Exact Geometrical Optics Scattering by a Right-Angle Wedge Made of Double-Negative Material," IEEE Transactions on Antennas and Propagation, 54, 8, August 2006, pp. 2301-2304. 
9. J. Van Bladel, Singular Electromagnetic Fields and Sources, chapter 4, Oxford, UK, Oxford University Press, 1991.

10. R. D. Graglia and A. F. Peterson, Higher-Order Techniques in Computational Electromagnetics, section 7.1, Edison, NJ, SciTech Publishing (IET), 2015.

11. P. Y. Ufimtsev, B. Khayatian, and Y. Rahmat-Samii, "Singular Edge Behavior: To Impose or Not ImposeThat Is the Question," Microwave and Optical Technology Letters, 24, 4, February 2000, pp. 218-223.

12. R. W. Ziolkowski and E. Heyman, "Wave Propagation in
Media Having Negative Permittivity and Permeability," Physical Review E, 64, October 2001, 056625, doi: 10. 1103/PhysRevE.64.056625.

13. P. L. E. Uslenghi, "Electromagnetic Scattering by Metallic Cylinders Perpendicularly Truncated by a Metal Plane," IEEE Transactions on Antennas and Propagation, 63, 5, May 2015, pp. 2228-2236.

14. P. L. E. Uslenghi, "Polarization Decoupling for Oblique Scattering by Penetrable Cylinders of Arbitrary Cross Section," IEEE Transactions on Antennas and Propagation, 65, 6, June 2017, pp. 3273-3274. 